\title{
ON A CLASS OF ANALYTIC FUNCTIONS INVOLVING THE SALAGEAN DIFFERENTIAL OPERATOR
}

\author{
S. ABDUL HALIM
}

\begin{abstract}
A bstract. We introduce the class $B_{n}(\alpha)$ consisting of functions analytic in the unit disc. In this paper, we give some properties of this class as well as considering some integral operators.
\end{abstract}

\section{Introduction}

Let $A$ be the calss of functions $f$ analytic in the unit disc $D=\{z:|z|<1\}$ and of the form

$$
f(z)=z+\sum_{j=2}^{\infty} a_{j} z^{j} .
$$

For a function $f \in A$, we consider the differential operator $D^{n}$ introduced by Salagean [4].

Definition 1.1. Let $f \in A$. For $n \geq 1$, define $D^{n}$ by

$$
\begin{aligned}
& D^{1} f(z)=D f(z)=z f^{\prime}(z) \\
& D^{n} f(z)=D\left(D^{n-1} f(z)\right)=z\left[D^{n-1} f(z)\right]^{\prime},
\end{aligned}
$$

where $D^{\circ} f(z)=f(z)$.

With the above definition, we now introduce the subclass $B_{n}(\alpha)$ of $A$ as follows:-

Received March 4, 1991. 
Defintion 1.2. For $\alpha>0$ and $n=0,1,2, \ldots$, a function $f$ normalized by (1.1) is said to be in $B_{n}(\alpha)$, if, and only if,

$$
\operatorname{Re} \frac{D^{n}\left[f(z)^{\alpha}\right]}{z^{\alpha}}>0
$$

for $z \in D$. (Powers in (1.3) are meant as principal values).

In the case $n=1, B_{1}(\alpha)$ denotes the class of Bazilevic functions with logarithmic growth. (see [5] and [6]). Earlier, in [2] it was proven that $B_{1}(\alpha) \subset S$, where $S$ is the subclass of $A$, consisting of univalent functions in $D$. Also let $P$ denote the class of analytic functions $p$ such that $p(0)=1$ and $\operatorname{Re} p(z)>0$ for $Z \in D$. In this paper, we give some properties of the class $B_{n}(\alpha)$ and consider an iterated integral operator problem.

\section{Preliminary Lemmas.}

We shall need the following lemmas.

Lemma 2.1. [3]. Let $M$ and $N$ be analytic in $D$ with $M(0)=N(0)=0$. If $N(z)$ maps $D$ onto a many sheeted region which is starlike with respect to the origin and $\operatorname{Re} \frac{M^{\prime}(z)}{N^{\prime}(z)}>0$ in $D$, then Re $\frac{M(z)}{N(z)}>0$ in $D$.

Lemma 2.2. Let $p(z)=1+\sum_{i=1}^{\infty} c_{i} z^{i} \in P$. Then for $\alpha+c>0$,

$$
q(z)=1+(\alpha+c) \sum_{i=1}^{\infty} \frac{c_{i} z^{i}}{(a+c+i)}, \quad z \in D
$$

in also in $P$.

Proof. Since $p \in P$, we then have

$$
\operatorname{Re}\left[\frac{z^{\alpha+c-1}+\sum_{i=1}^{\infty} c_{i} z^{i+\alpha+c-1}}{z^{\alpha+c-1}}\right]>0 .
$$

Fhus

$$
\operatorname{Re}\left[\frac{\left[\frac{z^{\alpha+c}}{\alpha+c}+\sum_{i=1}^{\infty} \frac{c_{i} z^{i+\alpha+c}}{\alpha+c+i}\right]^{\prime}}{\left[\frac{z^{\alpha+c}}{\alpha+c}\right]^{\prime}}\right]>0
$$


which by Lemma 2.1 above, gives our required result.

3. Some properties of $B_{n}(\alpha)$.

Theorem 3.1. $B_{n+1}(\alpha) \subset B_{n}(\alpha)$ for $n \geq 1$.

Proof. Let $f \in B_{n+1}(\alpha)$, then by (1.3), we have

$$
\operatorname{Re} \frac{D^{n+1} f(z)^{\alpha}}{z^{\alpha}}>0
$$

and so (1.2) gives

$$
\operatorname{Re} \frac{\alpha\left[D^{n} f(z)^{\alpha}\right]^{\prime}}{\left(z^{\alpha}\right)^{\prime}}>0
$$

which by Lemma 2.1 above, implies that

$$
\operatorname{Re} \frac{D^{n} \int(z)^{\alpha}}{z^{\alpha}}>0
$$

Hence $f \in B_{n}(\alpha)$.

Corollary. $B_{n}(\alpha) \subset S$ for $n=1,2,3, \ldots$

Proof. This is easily seen, since $B_{n}(\alpha) \subset B_{1}(\alpha)$ for $n=2,3,4, \ldots$ and $B_{1}(\alpha) \subset S$.

Theorem 3.2. Let $f \in B_{n}(\alpha)$ and $\alpha+c>0$. Then the function $F$ defined by

$$
F(z)^{\alpha}=\frac{\alpha+c}{z^{c}} \int_{0}^{z} t^{c-1} f(l)^{\alpha} d t, \quad(z \in D)
$$

is also in the class $B_{n}(\alpha)$.

Proof. Since $f \in B_{n}(\alpha)$, there exists $p \in P$ with $p(z)=1+\sum_{i=1}^{\infty} c_{i} z^{i}$ such that

$$
\frac{D^{n} f(z)^{\alpha}}{z^{\alpha}}=\alpha^{n} p(z) .
$$

(1.2) and (3.2) give

$$
\frac{D^{n-1} f(z)^{\alpha}}{z^{\alpha}}=\alpha^{n-1}\left[1+\alpha \sum_{j=1}^{\infty} \frac{c_{j} z^{j}}{(j+\alpha)}\right] .
$$


Now, on assumption that

$$
\frac{D^{n-k} f(z)^{\alpha}}{z^{\alpha}}=\alpha^{n-k}\left[1+\alpha^{k} \sum_{j=1}^{\infty} \frac{c_{j} z^{j}}{(j+\alpha)^{k}}\right]
$$

is true for some $0<k \leq n$, we have

$$
\left[\frac{f(z)}{z}\right]^{\alpha}=1+\alpha^{n} \sum_{j=1}^{\infty} \frac{c_{j} z^{j}}{(j+\alpha)^{n}}
$$

which follows trivally by induction.

Using (3.3) in (3.1) gives

$$
\left[\frac{F(z)}{z}\right]^{\alpha}=1+(\alpha+c) \sum_{j=1}^{\infty} \frac{\alpha^{n} c_{j} z^{j}}{(j+\alpha)^{n}(j+\alpha+c)}
$$

Next, assume that

$$
\alpha^{-k} \frac{D^{k} F(z)^{\alpha}}{z^{\alpha}}=1+\alpha^{n-k}(\alpha+c) \sum_{j=1}^{\infty} \frac{c_{j} z^{j}}{(j+\alpha)^{n-k}(j+\alpha+c)}
$$

is true for $0<k \leq n$.

-Hence

$$
\alpha^{-n} \frac{D^{n} F(z)^{\alpha}}{z^{\alpha}}=1+(\alpha+c) \sum_{j=1}^{\infty} \frac{c_{j} z^{j}}{(j+\alpha+c)}
$$

follows by induction.

Finally, since $p \in P$, by Lemma 2.2 we have

$$
\operatorname{Re} \frac{D^{n} F(z)^{\alpha}}{z^{\alpha}}>0
$$

i.e. $F \in B_{n}(\alpha)$.

Theorem 3.3. Let $f \in B_{n}\left(\alpha^{\prime}\right)$. Then for $n \geq 0$ and $z=r e^{i \theta} \in D$,

$$
1+2 \alpha^{n} \sum_{j=1}^{\infty} \frac{(-r)^{j}}{(j+\alpha)^{n}} \leq \operatorname{Re}\left[\frac{f(z)}{z}\right]^{\alpha} \leq\left|\frac{f(z)}{z}\right|^{\alpha} \leq 1+2 \alpha^{n} \sum_{j=1}^{\infty} \frac{r^{j}}{(j+\alpha)^{n}}
$$


The result is sharp.

Proof.

(i) We first prove the lower bound.

From (3.2), since $p \in P$, we have

$$
\alpha^{-n} \operatorname{Re} \frac{D^{n} f(z)^{\alpha}}{z^{\alpha}}=\operatorname{Re} p(z) \geq \frac{1-r}{1+r}=1+2 \sum_{j=1}^{\infty}(-r)^{j} .
$$

Next, for some $0<k \leq n$, assume that

$$
\alpha^{-k} \operatorname{Re} \frac{D^{k} f(z)^{\alpha}}{z^{\alpha}} \geq 1+2 \alpha^{n-k} \sum_{j=1}^{\infty} \frac{(-r)^{j}}{(j+. \alpha)^{n-k}}
$$

Then (1.2) gives

$$
\begin{aligned}
\alpha^{1-k} \operatorname{Re} \frac{D^{k-1} f(z)^{\alpha}}{z^{\alpha}} & =\alpha^{1-k} \operatorname{Re} \frac{1}{z^{\alpha}} \int_{0}^{z}\left[D^{k-1} f(t)^{\alpha}\right]^{\prime} d t \\
& =\frac{\alpha^{1-k}}{r^{\alpha}} \int_{0}^{r} \rho^{\alpha-1} \operatorname{Re} \frac{D^{k} f\left(\rho e^{i \theta}\right)^{\alpha}}{\left.(\rho e)^{i \theta}\right)^{\alpha}} d \rho, \text { where } t=\rho e^{i \theta} \\
& \geq \frac{\alpha^{1-k}}{r^{\alpha}} \int_{0}^{r} \rho^{\alpha-1}\left[\alpha^{k}+2 \alpha^{n} \sum_{j=1}^{\infty} \frac{(-\rho)^{j}}{(j+\alpha)^{n-k}} d \rho,\right.
\end{aligned}
$$

on using (3.4).

Hence the lower bound follows by induction.

(ii) We next prove the upper bound.

Again, we use induction. Obviously, due to $|p(z)| \leq \frac{1+r}{1-r}$, we have from $(3.2)$,

$$
\alpha^{-n}\left|\frac{D^{n} f(z)^{\alpha}}{z^{\alpha}}\right| \leq \frac{1+r}{1-r}=1+2 \sum_{j=1}^{\infty} r^{j}
$$

For fixed $n$, assume that when $0<k \leq n$,

$$
\alpha^{-k}\left|\frac{D^{k} f(z)^{\alpha}}{z^{\alpha}}\right| \leq 1+2 \alpha^{n-k} \sum_{j=1}^{\infty} \frac{r^{j}}{(j+\alpha)^{n-k}}
$$


Then

$$
\begin{aligned}
\alpha^{1-k}\left|\frac{D^{k-1} f(z)^{\alpha}}{z^{\alpha}}\right| & \leq \frac{\alpha^{1-k}}{r^{\alpha}} \int_{0}^{r} \rho^{\alpha-1}\left|\frac{D^{k} f\left(\rho e^{i \theta}\right)^{\alpha}}{\left(\rho e^{i \theta}\right)^{\alpha}}\right| d \rho \\
& \leq 1+2 \alpha^{n-(k-1)} \sum_{j=1}^{\infty} \frac{r^{j}}{(j+\alpha)^{n-k+1}} .
\end{aligned}
$$

The proof now follows by induction. For fixed $n$, the result is sharp, since the upper and lower bounds for $\operatorname{Re} p(z)$ and $|p(z)|$ are sharp.

4. Iterated integral operator.

In [1], the authors gave the sharp estimate for the lower bound of $\operatorname{Re}(f(z) /$ $z)^{\alpha}$ when $f \in B_{1}(\alpha)$. This estimate can be seen in Theorem 3.3 in the case $n=1$. This result was then extended to include iterated integrals.

We now will give the sharp result for a more generalized version of iterated integral operator. First, for $z \in D, a>-1$ and $m=1,2,3, \ldots$ let

$$
I_{m}(z)=\frac{a+1}{z^{a+1}} \int_{0}^{z} t^{a} I_{m-1}(t) d t
$$

where $I_{0}(z)=(f(z) / z)^{\alpha}$.

Theorem 4.1. For a fixed $n$, let $f \in B_{n}(\alpha)$. Then for $z=r e^{i \theta} \in D$ and $m \geq 0$,

$$
\operatorname{Re} I_{m}(z) \geq \gamma_{m}(r)
$$

and

$$
\gamma_{m}(r)<1
$$

where for $m=0,1,2, \ldots$

$$
\gamma_{m}(r)=1+2 \alpha^{n}(1+a)^{m} \sum_{j=1}^{\infty} \frac{(-r)^{j}}{(j+\alpha)^{n}(j+a+1)^{m}} .
$$

Equality is attained for $f$ where $\left[\frac{D^{n} f(z)^{\alpha}}{z^{\alpha}}\right]=\alpha^{n}\left[\frac{1-z}{1+z}\right]$. 
Proof. From Theorem 3.3, we have

$$
\operatorname{Re} I_{0}(z) \geq 1+2 \alpha^{n} \sum_{j=1}^{\infty} \frac{(-r)^{j}}{(j+\alpha)^{n}} \text {. }
$$

Next, from (4.1)

$$
\begin{aligned}
\operatorname{Re} I_{k+1}(z) & =\operatorname{Re} \frac{a+1}{z^{a+1}} \int_{0}^{z} t^{a} I_{k}(t) d t \\
& =\frac{a+1}{r^{a+1}} \int_{0}^{r} \rho^{a} \operatorname{Re} I_{k}\left(\rho e^{i \theta}\right) d \rho \\
& \geq \frac{a+1}{r^{a+1}} \int_{0}^{r} \rho^{a}\left[1+2 \alpha^{n}(1+a)^{k} \sum_{j=1}^{\infty} \frac{(-\rho)^{j}}{(j+\alpha)^{n}(j+a+1)^{k}}\right] d \rho \\
& =1+2 \alpha^{n}(1+a)^{k+1} \sum_{j=1}^{\infty} \frac{(-r)^{j}}{(j+\alpha)^{n}(j+a+1)^{k+1}} \\
& =\gamma_{k+1}(r) .
\end{aligned}
$$

Hence, the first inequality follows by induction.

Now, for $m \geq 0$, the series

$$
\gamma_{m}(r)=1+2 \alpha^{n}(1+a)^{m} \sum_{j=1}^{\infty} \frac{(-r)^{j}}{(j+\alpha)^{n}(j+a+1)^{m}}
$$

is absolutely convergent. On rearranging pairs of terms in $\gamma_{m}(r)$ as

$$
\begin{aligned}
& \gamma_{m}(r) \\
= & 1-2 \alpha^{n}(1+a)^{m}\left[\frac{r}{(1+\alpha)^{n}(2+a)^{m}}-\frac{r^{2}}{(2+\alpha)^{n}(3+a)^{m}}\right] \\
& -2 \alpha^{n}(1+a)^{m}\left[\frac{r^{3}}{(3+\alpha)^{n}(4+a)^{m}}-\frac{r^{4}}{(4+\alpha)^{n}(5+a)^{m}}\right]+\ldots \\
= & 1-2 \alpha^{n}(1+a)^{m} \sum_{j=1}^{\infty}\left[\frac{r^{2 k-1}}{(2 k-1+\alpha)^{n}(2 k+a)^{m}}-\frac{r^{2 k}}{(2 k+\alpha)^{n}(2 k+1+a)^{m}}\right]
\end{aligned}
$$

shows that $\gamma_{m}(r)<1$.

This completes the proof. 
Remark. Finally, we conjecture that for $\gamma_{m}(r)$ given by (4.2), it also satisfies $\gamma_{m}(r)>\gamma_{m}(1)$.

\section{Acknowledgement}

I would like to thank the referee for his useful suggestions.

\section{References}

[1] S. Abdul Halim and D. K. Thomas, "A Note on Bazilevic Functions", Internat. J. Math. and Math. Sci. To appear

[2] I. E. Bazilevic, "On a case of integrability in quadratures of the Loewner - Kufarev equation", Math. Sb. N. S. 37 (1955), pp.471-476.

[3] R. J. Libera, "Some classes of regular Univalent functions", Proc. Amer. Math. Soc. 16 (1965), pp. 755-758.

[4] G. S. Salagean, "Subclasses of univalent functions", Lecture notes in Math 1013. (1983), pp. 362-372.

[5] R. Singh, "On Bazilevic functions", Proc. Amer. Math. Soc. 38(2) (1973), pp. 261-271.

[6] D. K. Thomas, "On a subclass of Bazilevic functions", Internat. J. Math. and Math. sci. 8(4) (1985), pp. 779-783.

Department of Mathematics, University of Malaya, 59100, Kuala Lumpur, Malaysia. 